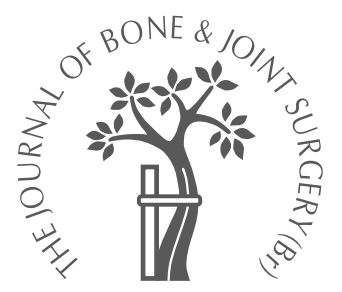

J. R. Robinson, J. Sanchez-Ballester, A. M. J. Bull, R. de W.M.Thomas, A. A. Amis

From Imperial College, London, England

\title{
The posteromedial corner revisited
}

\author{
AN ANATOMICAL DESCRIPTION OF THE PASSIVE RESTRAINING \\ STRUCTURES OF THE MEDIAL ASPECT OF THE HUMAN KNEE
}

We have reviewed the literature on the anatomy of the posteromedial peripheral ligamentous structures of the knee and found differing descriptions. Our aim was to clarify the differing descriptions with a simplified interpretation of the anatomy and its contribution to the stability of the knee.

We dissected 20 fresh-frozen cadaver knees and the anatomy was recorded using video and still digital photography. The anatomy was described by dividing the medial collateral ligament (MCL) complex into thirds, from anterior to posterior and into superficial and deep layers. The main passive restraining structures of the posteromedial aspect of the knee were found to be superficial MCL (parallel, longitudinal fibres), the deep MCL and the posteromedial capsule (PMC). In the posterior third, the superficial and deep layers blend. Although there are oblique fibres (capsular condensations) running posterodistally from femur to tibia, no discrete ligament was seen. In extension, the PMC appears to be an important functional unit in restraining tibial internal rotation and valgus.

Our aim was to clarify and possibly simplify the anatomy of the posteromedial structures. The information would serve as the basis for future biomechanical studies to investigate the contribution of the posteromedial structures to joint stability.

\begin{abstract}
J. R. Robinson, MRCS, Clinical Research Fellow J. Sanchez-Ballester FRCS (Ed), Clinical Research Fellow

A. A. Amis, DSc, Professor of Orthopaedic

Biomechanics

Biomechanics Section

Department of Mechanical Engineering

A. M. J. Bull, PhD, ARC

Lecturer in Bioengineering Department of Biology Imperial College of Science, Technology and Medicine, Exhibition Road, London SW7 2AZ, UK.

R. de W. M. Thomas, MS, FRCS, Consultant Orthopaedic Surgeon Department of

Musculoskeletal Surgery, Imperial College London, Charing Cross Hospital,

Fulham Palace Road, London W6 8RF, UK.

Reconstructions of the anterior and posterior cruciate ligaments are routinely undertaken. Rupture of a cruciate ligament can occur in association with other ligamentous injuries. ${ }^{1,2}$ There is controversy about the management of the resulting complex instability and increasing interest in the importance of the peripheral structures and the associated rotational instability which may be caused by injury to restraining structures at either the posteromedial or posterolateral corners.

In order to improve the diagnosis of complex ligamentous injuries and allow rational surgical treatment, a better understanding of the medial structures of the knee is needed. There is some confusion in the literature about the anatomical detail and function of the medial ligament complex.
\end{abstract}

Correspondence should be sent to Professor A. A. Amis.

(C)2004 British Editorial Society of Bone and Joint Surgery doi:10.1302/0301-620X.86B5. $14853 \$ 2.00$

$J$ Bone Joint Surg $[\mathrm{Br}]$ 2004;86-B:674-81.

Received 11 August 2002;

Accepted after revision

21 January 2004

\section{Literature review}

Studies by Palmer $^{3}$ (Fig. 1) and Brantigan and Voshell $^{4}$ (Fig. 2) provided early, detailed, anatomical descriptions and their work is often cited. More recent descriptions vary, some being over simplifications with others unduly complex. Some previous authors used artists' sketches which give differing impressions of the same structure; the artist's interpretation rather than the more subtle anatomy encountered by the surgeon attempting reconstruction.

After Palmer's original work, ${ }^{3}$ most authors recognised that the medial collateral ligament (MCL) has superficial and deep parts. The most detailed exposition of this "layered approach" was by Warren and Marshall ${ }^{5}$ who described three distinct layers: fascia, superficial and deep MCL.

The superficial MCL. The femoral attachment has been variously described as being just anterior to,${ }^{6}$ distal to, ${ }^{7,8}$ or $10 \mathrm{~mm}$ anterior and distal to ${ }^{9}$ the adductor tubercle, and forming an oval $20 \mathrm{~mm}$ high, with its greatest diameter in the long axis of the femur. ${ }^{3}$ The tibial attachment has been described as lying between a mean of $46 \mathrm{~mm}^{4}$ and $60 \mathrm{~mm}^{3}$ distal to the joint or one handbreadth, according to Last; ${ }^{7}$ the length of the ligament being between 90 and $110 \mathrm{~mm} \cdot{ }^{10}$ At its tibial attachment, the fibres blend tangentially with the periosteum. The superficial MCL has been found to be $10 \mathrm{~mm}$ wide proximally, increasing to $20 \mathrm{~mm}$ at the joint line. Its width decreases distally and, just proximal to its insertion, the fibres are bridged by the pes anserinus. ${ }^{3,6}$ It is consistently described as being made up of parallel and 
oblique fibres. The parallel ones run proximodistally and have an easily identifiable anterior border. ${ }^{6,11}$ At the joint line the width of this part of the ligament varies from 10 $\mathrm{mm}$ to $14 \mathrm{~mm}$ and these fibres have been shown to be separate from the capsule. ${ }^{7}$

Immediately behind the parallel fibres are posterior oblique fibres forming a "triangular membrane reinforcing the posterior capsule". ${ }^{12}$ From the femoral attachment proximally, the fibres run obliquely backwards to the joint line. Thereafter they run distally and anteriorly to the tibia. ${ }^{4,12}$ Warren and Marshall ${ }^{5}$ showed them to be in continuity with the posterior edge of the parallel fibres. Brantigan and Voshell ${ }^{4}$ showed that the parallel fibres were distinct from the capsule but the oblique fibres were inseparable. Last ${ }^{7}$ found that the fibres running posterodistally were fused with the capsule proximal to the joint line, but those fibres running distal to the joint line were separated from the capsule by an expansion of the semimembranosus tendon. Palmer ${ }^{3}$ also recognised proximal and distal oblique fibres in the deep part of the ligament, lying behind the long superficial fibres and attached to the medial meniscus. They prevented posterior movement of the meniscus in flexion. Slocum and Larson ${ }^{12}$ and Brantigan and Voshell ${ }^{4}$ described a fan-like sheet of tissue extending anteriorly from the anterior border of the MCL to the patella and blending with its retinaculum (Fig. 2).

The effect of flexion and extension of the knee on the superficial MCL. Several authors have contrasted the appearance of the superficial MCL in flexion and extension. Some described it as a single entity: Last $^{7}$ stated that because its femoral attachment was posterior to the axis of rotation of the femoral condyles, it was relaxed in flexion and taut in extension. Mains et $a 1,{ }^{6}$ stated that because the femoral attachment originates near the centre of rotation of the knee, the tension remains constant throughout flexion. Others have described the appearance of different parts of the ligament: Horwitz, ${ }^{13}$ describing it as the internal collateral ligament, found it taut in extension and, in flexion, the posterior part relaxed while the anterior part remained tense. Warren et $\mathrm{al}^{11}$ also noticed the same phenomenon. Others have stated that during flexion the posterior expansions lose little of their tension. ${ }^{10}$ Palmer $^{3}$ noted that the upright oval femoral attachment, parallel to the long axis of the femur in extension, became progressively more horizontal, until, at $90^{\circ}$ of flexion it reached its greatest width. He therefore concluded that the ligament did not relax in its entirety throughout flexion. The anterior part became tense in flexion and the posterior longitudinal fibres tense in extension. Although the schematic diagrams of Warren et $\mathrm{al}^{11}$ show the femoral attachment as wider in extension and narrower in flexion, they found a similar pattern of fibre recruitment during flexion and extension.

The deep MCL. The femoral attachment was described by Mains et $\mathrm{al}^{6}$ as lying just anterior and distal to the attachment of the superficial MCL, and just anterior to the more superficial tibial collateral ligament by Slocum and

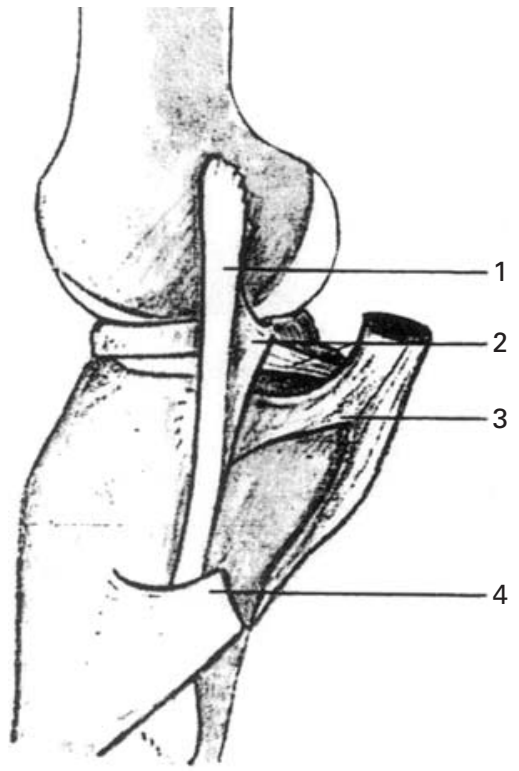

Fig. 1

A diagram showing Palmer's ${ }^{3}$ description of the superficial MCL (reproduced with permission from Palmer I. On the injuries to the ligaments of the knee joint. Acta Orthop Scand (Suppl) 1938:5-283).

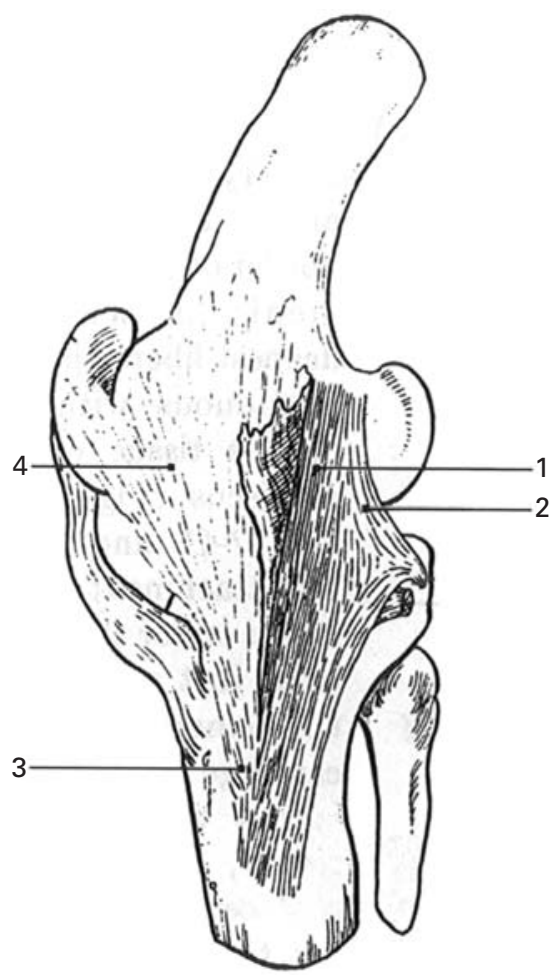

Fig. 2

A diagram showing Brantigan and Voshell's ${ }^{4}$ description of the medial aspect of the knee: 1 , parallel portion of $\mathrm{MCL} ; 2$, oblique portion of $\mathrm{MCL} ; 3$, the area on the tibia where the fibrous fan-like membrane ${ }^{4}$ is indistinguishable from the attachment of the MCL; 4, fibrous fan-like membrane (reproduced with permission from Brantigan OC, Voshell AF. The tibial collateral ligament: its function to the medial mensicus. $J$ Bone Joint Surg 1943;25:121-31). 


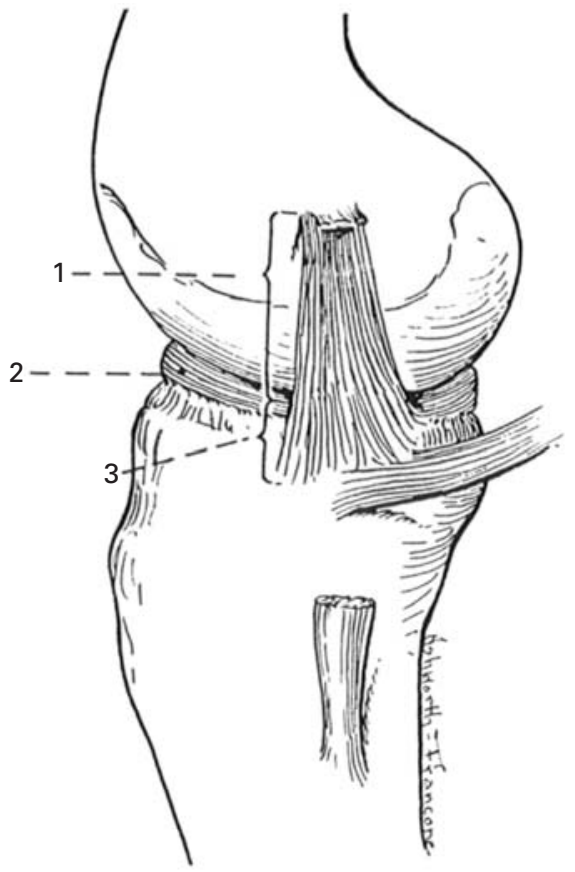

Fig. 3

A diagram showing Slocum and Larson's ${ }^{12}$ deep layer of the MCL: 1, meniscofemoral portion; 2, medial meniscus; 3, meniscotibial portion (reproduced with permission from Slocum DB, Larson $\mathrm{RL}$. Rotary instability of the knee: its pathogenesis and a clinical test to determine its presence. J Bone Joint Surg [Am] 1968:50-A:211-25).

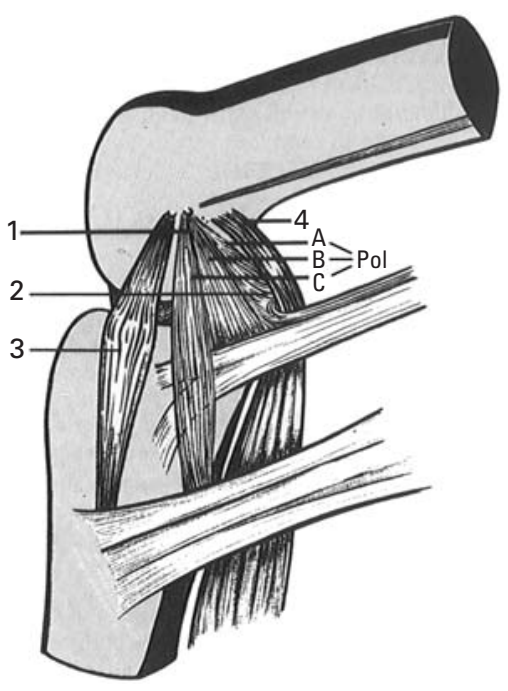

Fig. 4

A diagram showing Hughston and Eiler's ${ }^{9}$ description of the posteromedial corner of the knee: 1, the common ligament of origin of the posterior oblique ligament (POL); 2 , the oblique popliteal ligament; 3 , the tibial collateral ligament; 4 , the medial head of gastrocnemius. The POL with A) superior or capsular arm; B) central or tibial arm C) superficial arm which passes over semimembranosus (reproduced with permission from Hughston JC, Eilers AF. The role of the posterior oblique ligament in repairs of acute medial collateral ligament tears of the knee. J Bone Joint Surg [Am] 1973;55-A;923-40).
Larson $^{12}$ (Fig. 3). Most authors agree that the deep part is attached to the medial meniscus as meniscofemoral and meniscotibial portions. The deep MCL was described by Slocum and Larson ${ }^{12}$ as attaching to the tibia just below its articular surface. Last $^{7}$ described its attachment to the articular margin of the tibia, but that its femoral attachment was to the articular margin of the femur. This attachment would limit movement of the joint. Last ${ }^{7}$ noted that, despite the significance of the structure, it had no official name.

There is disagreement as to the differentiation of the deep MCL from the capsule. $\mathrm{Last}^{7}$ described the deep MCL as being continuous with the rest of the capsule whilst Hughston and Eilers ${ }^{9}$ found that the deep part of the MCL blended with medial capsular fibres in some knees but was separate in others and therefore not attached to the meniscus. Warren and Marshall ${ }^{5}$ found that the meniscofemoral portion of the deep MCL was a discrete structure in only some knees, having a separate attachment from the superficial ligament approximately $5 \mathrm{~mm}$ distal to it. Slocum and Larson ${ }^{12}$ described the capsule as having distinct anterior, middle and posterior parts with their middle capsular ligament corresponding to the deep MCL. They described the deep part of the ligament as lying beneath the superficial MCL, yet Policau Prose, Lohman and Huson ${ }^{14}$ described the deep or meniscal part of the MCL as lying posterior to the superficial femorotibial part of the ligament. The fibres of the ligament have been variously described as running posteroinferiorly from the femur to the tibia, ${ }^{3}$ anteroinferiorly ${ }^{5}$ or in a fan-like manner. ${ }^{6}$

The effect of flexion and extension of the knee on the deep MCL. The fibres have been described as being tight in extension ${ }^{15}$ and slackening as the knee flexes, due to the shape of the medial femoral condyle and recession of the medial meniscus. ${ }^{10}$

The posteromedial capsule (PMC). There are differing descriptions of the oblique fibres running distally and posteriorly from their femoral attachment to the posteromedial aspect of the tibia. Brantigan and Voshell ${ }^{4}$ and Williams et $\mathrm{al}^{8}$ described them as part of the posterior fibre bundles of the superficial MCL. Others described a distinct posterioroblique ligament (POL), separate from the superficial $\mathrm{MCL}^{9,14,16}$ (Fig. 4). This ligament is said to be attached to the posterior aspect of the medial meniscus and inseparable from the capsule. Its femoral attachment has been described as from the adductor tubercle, ${ }^{9}$ the same attachment as the superficial MCL, ${ }^{6}$ or from an area between the adductor tubercle and the medial epicondyle. ${ }^{14}$ The POL has been said to attach to the posteromedial corner of the tibia immediately proximal to the insertion of the semimembranosus, with a portion of the ligament swinging anterodistally over this tendon. ${ }^{6}$ The oblique capsular thickening of the MCL, as described by Williams et $\mathrm{al},{ }^{8}$ with fibres running from the medial femoral condyle to the posterior part of the tibia, corresponds to the "central part" of the POL described by Hughston and Eilers. ${ }^{9}$ Williams et al, ${ }^{8}$ however, did not differentiate between the superficial and deep 


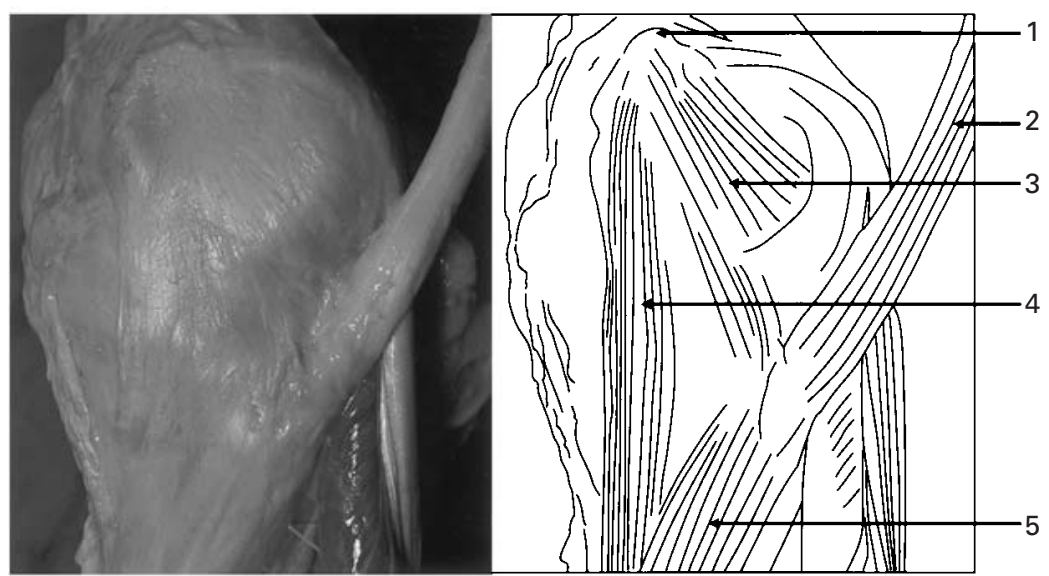

\section{Fig. 5}

Photograph and diagram describing layer 2, the layer of the superficial MCL. Medial aspect of right knee, anterior to left: 1, Adductor tubercle; 2, Semimembranosus; 3, PMC enveloping the posterior medial femoral condyle; 4, Longitudinal fibres of superficial $\mathrm{MCL} ; 5$, Distal oblique fibres of superficial MCL. parts of the MCL. Fischer et $\mathrm{al}^{17}$ also studied the anatomical relationships of the POL and showed a direct anatomical relationship between it and the long fibres of the superficial MCL. However, they were unable using their method, to show a functional relationship and concluded that the POL and MCL must work independently. ${ }^{17}$

The intimate relationship of the semimembranosus tendon to the posteromedial corner has been noted and described differently in several anatomical studies. Hughston and Eilers ${ }^{9}$ described the POL as having three distinct arms: a "capsular arm" running deep to gastrocnemius but joining the upper fibres or "popliteal arm" of the semimembranosus tendon to form the oblique popliteal ligament; a "central arm" attaching to the tibia beneath the semimembranosus tendon; and a "superficial arm", passing over the semimembranosus tendon and blending with the thick semimembranosus sheath. However, they believed this distal arm to be functionally insignificant. Policau Prose et al, ${ }^{14}$ described a POL blending with the medial head of gastrocnemius and a proximal expansion of the semimembranosus tendon. Warren and Marshall ${ }^{5}$ described both the semimembranosus tendon sheath and the tendon as blending with the posteromedial corner and forming the oblique popliteal ligament from its posterior fibres. The tendon, however, was described as not participating in any of the layers of the MCL as it inserts directly to bone. Kaplan ${ }^{18}$ found that the tendinous portion of the muscle divided at the joint line into three tendo-ligamentous structures anteromedial to the medial head of gastrocnemius. He, in contrast to Warren and Marshall, ${ }^{5}$ described two insertions, an anterior lying deep to the superficial MCL and a posterior, lying just behind the insertion of the deep MCL. Last $^{7}$ referred also to the varying accounts of the anatomy of the semimembranosus tendon with descriptions of an expansion of the tendon attached to the medial meniscus.

The effect of flexion and extension of the knee on the posteromedial corner. As the knee flexes, Strobel and Stedfeld ${ }^{10}$ showed the POL "winding up". They stated that the POL was "self-tightening" during flexion. ${ }^{10}$ Hughston and Eilers ${ }^{9}$ found that, at $60^{\circ}$ of flexion it was slack and had no function as a static stabiliser. Yet, even at $30^{\circ}$ of flexion, they recorded a $16 \%$ reduction in the distance between femoral and tibial attachments from their position in extension, which means it was slack. However, they stated that a kinetic stabilising effect was produced by the ligaments through contraction of the semimembranosus.

\section{Methods}

We dissected 20 cadaver knees. Relatives were interviewed and signed a consent form, which had been approved by the Local Research Ethics Committee. The age range of specimens was 75 to 93 years with 12 left and eight right knees. Fresh, or fresh-frozen specimens allow better anatomical detail than specimens preserved in formalin. ${ }^{5}$ Within two days of death, the knees were extracted complete with surrounding subcutaneous soft tissue and approximately 20 $\mathrm{cm}$ of extracapsular femur and tibia, and frozen at $-20^{\circ} \mathrm{C}$ in sealed polythene bags to prevent dehydration. We performed dissections immediately after thawing. The femur and tibia were stripped of muscle and subcutaneous fat, taking care to preserve the ligaments and joint capsule intact. The medial ligament complex was carefully dissected, layer-by-layer. The pattern and definition of the structures were examined and recorded. Exposure of the structures deep to the superficial MCL was achieved by dividing the superficial MCL (parallel and inferior oblique fibres) close to its tibial attachment and folding it proximally. We noted whether the fibres of the posterior aspect of the MCL complex were distinguishable from the insertion of the semimembranosus tendon and if it was possible to clearly identify a POL.

We recorded the appearance of the MCL complex as the specimens were flexed, extended, and rotated using still and video digital photography. We studied how the ligaments appeared to act and in what positions different parts of the complex either tightened or relaxed. 


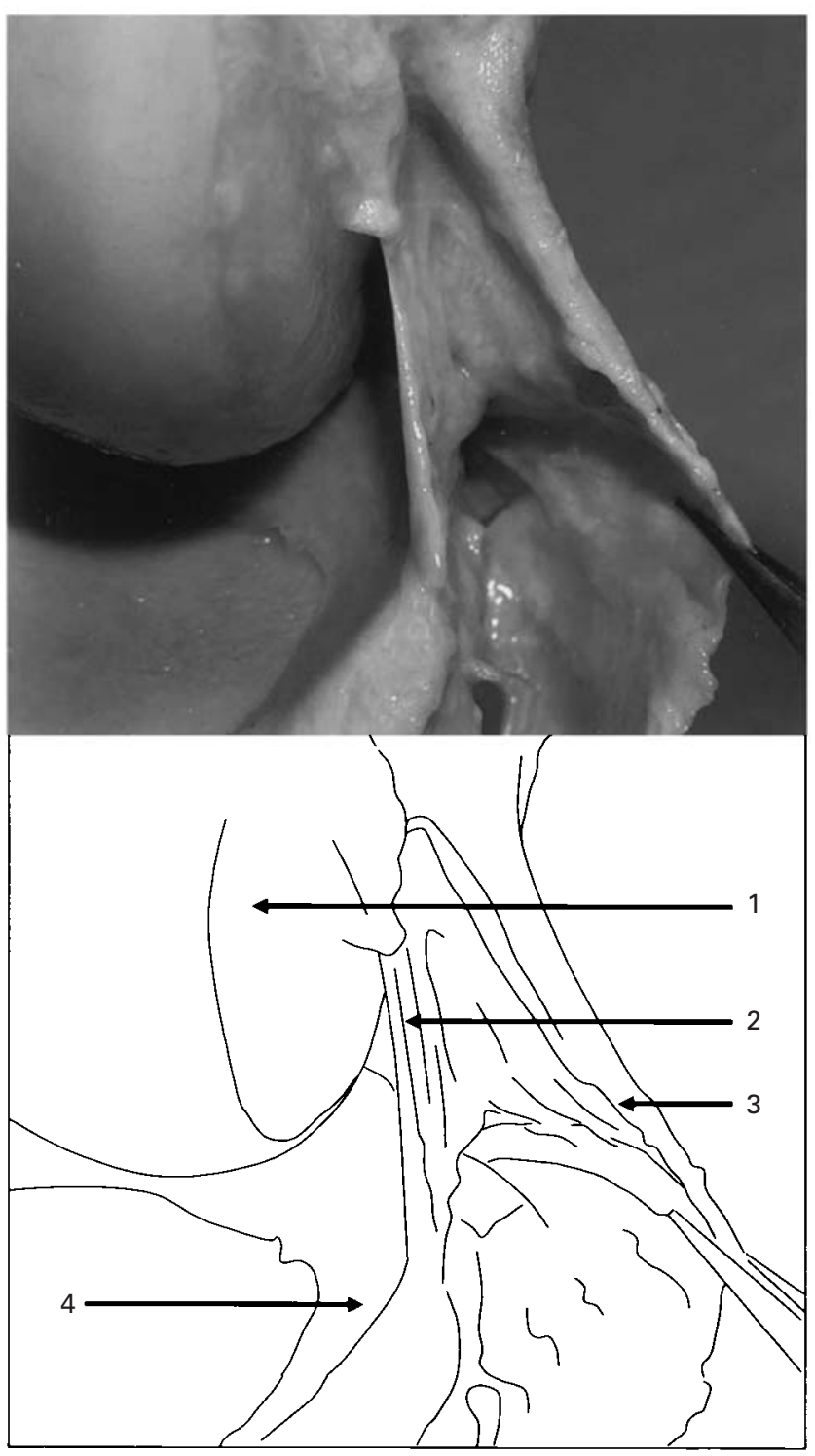

Fig. 6

Photograph and diagram describing the deep MCL, viewed in a right knee from anterior to posterior after removal of the superficial fascia and capsule anterior to the $\mathrm{MCL}$, and detachment of tibial attachment of superficial MCL: 1, medial femoral condyle; 2, deep MCL - femoromeniscal part; 3, superficial MCL elevated medially; 4, medial meniscus.

\section{Results}

We found that the easiest way to describe the medial aspect of the knee from the edge of the patellar tendon to the most medial edge of the medial head of gastrocnemius, was by dividing it into thirds from anterior to posterior (Fig. 5): 1) anterior: from the medial border of the patellar tendon to the anterior border of the longitudinal fibres of the superficial MCL; 2) middle: the width of the longitudinal fibres of the MCL; and 3) posterior: the posterior border of the longitudinal fibres of the superficial MCL to the medial head of gastrocnemius.
Our study of the posteromedial structures focused on the middle and posterior thirds. Also, each third was described in layers following Warren and Marshall's ${ }^{5}$ descriptions: layer 1, the fascial layer; layer 2, the superficial MCL; layer 3, the capsule or deep MCL (Figs 6 and 7).

Layer 1 was not considered in detail as Warren and Marshall ${ }^{5}$ have previously described it extensively. It covers the entire medial aspect of the knee and posteriorly is continuous with the deep fascia of the lower limb. As such it is not a structure specific to the MCL complex.

Anterior. There was no substantial ligament structure linking the femur to the tibia in the anterior third. In layer 1 of fascia, the fibres blended anteriorly and distally with the tibial periosteum at the level of the attachment of the superficial MCL. In layer 2, weak fibres from the anterior border of the superficial MCL appeared to blend with layer 1 . Although the anterior third was not the subject of this study, it was noted that layer 2 also contained patellar retinacular fibres. The transverse fibres of the medial patellofemoral ligament wrapped over the superficial MCL at the medial epicondyle (Fig. 7). In layer 3, the capsule attached to the medial femoral condyle, thereby leaving the area around the epicondyle as extracapsular.

Middle. Layer 1 is the fascial layer. The longitudinal parallel fibres of the superficial MCL make up layer 2. The majority of the fibres of the superficial MCL attached to the distal aspect of the medial epicondyle but the most superficial fibres enveloped the epicondyle. The fibres blended tangentially with the periosteum on the medial aspect of the tibia. The attachment lay obliquely on the tibia 60 to $80 \mathrm{~mm}$ distal to the joint line, forming an area 2 to $4 \mathrm{~mm}$ wide and 12 to $18 \mathrm{~mm}$ long, running from anterior to posterior and proximal to distal. There were clearly identifiable parallel fibres (of a length of 80 to $112 \mathrm{~mm}$ and width of 10 to 13 $\mathrm{mm}$ ). The anterior margin of this structure was clearly defined by its longitudinal fibres but posteriorly the margins were less clear. At the posterior margin of the longitudinal fibre band of the middle section, the longitudinal fibres blended with oblique fibres that formed part of the posterior third.

The superficial MCL appeared to remain taut as the knee was flexed. The more posterior fibres appeared to undergo greater changes of length with flexion and extension, becoming tighter as the knee extended. The longitudinal parallel fibres of the superficial MCL appeared to tighten further with external and internal rotation of the tibia (Fig. 8).

After transecting the superficial MCL (layer 2) with a transverse incision just proximal to the tibial attachment it was easy to fold it back to expose the deep MCL (layer 3), but the tibial attachment of the PMC (conjoined layers 2 and 3) prevented further reflection (Fig. 6).

The deep MCL is layer 3. The femoral attachment (layer 3) was oval approximately $7 \mathrm{~mm}$ in anteroposterior extent, 15 to $17 \mathrm{~mm}$ proximal to the femoral articular cartilage, immediately posterior and distal to the attachment of the superficial MCL and on the inferior surface of the epi- 


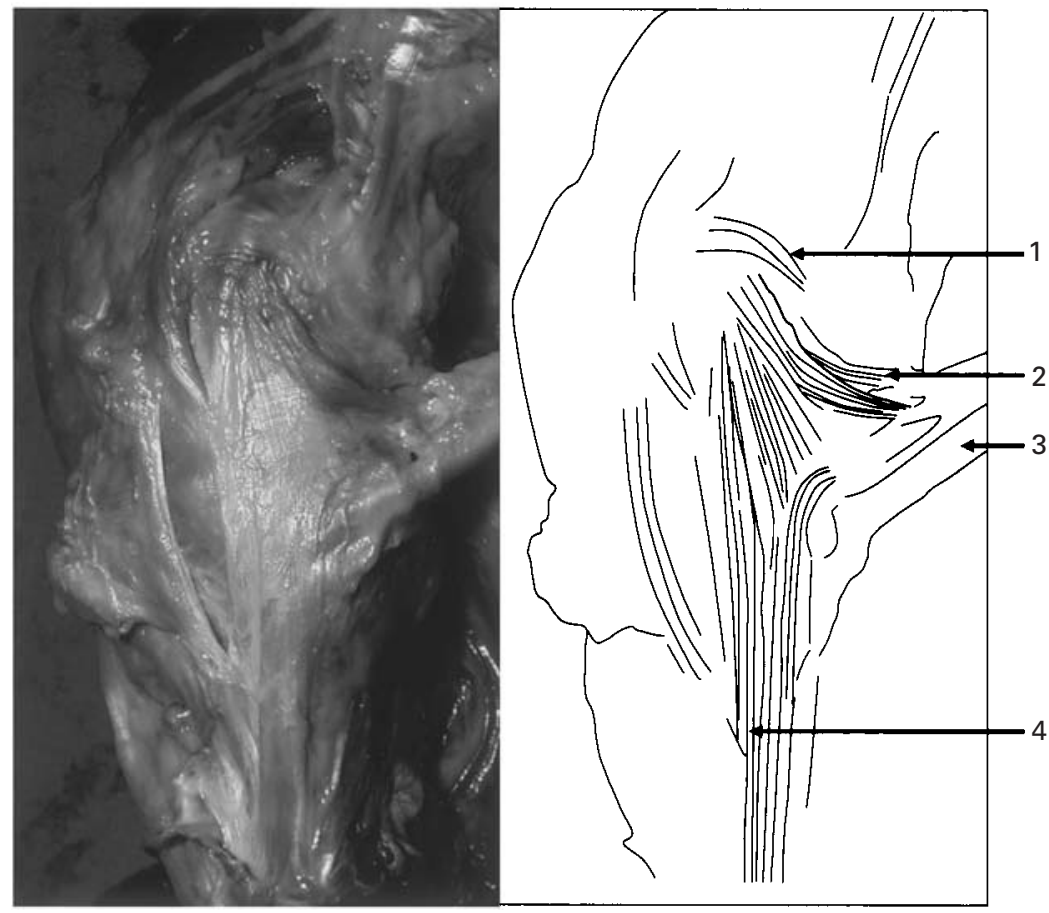

Fig. 7

Photograph and diagram describing the posteromedial corner in $30^{\circ}$ flexion. The medial aspect of the right knee, anterior to left: 1, patellofemoral ligament; 2, PMC, crumpling and folding behind itself as the knee flexes; 3, semimembranosus tendon; 4, longitudinal fibres of superficial MCL remain taut throughout flexion and extension, in valgus rotation at all angles of flexion.

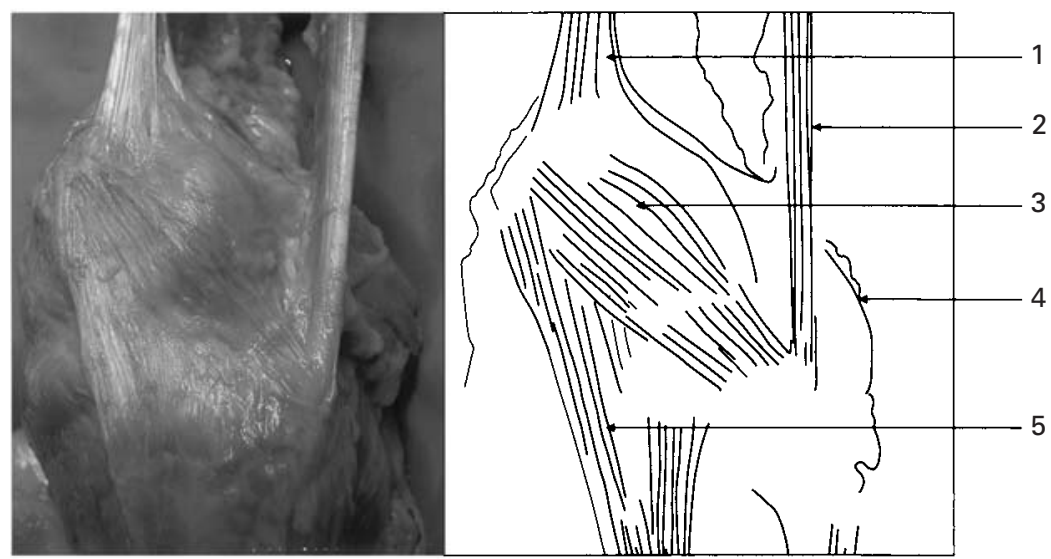

Fig. 8

Photograph and diagram describing the posteromedial corner on internal rotation in extension: 1 , adductor tendon; 2, semimembranosus; 3, fibres of PMC coming under tension as the tibia is internally rotated on femur; 4 , lateral femoral condyle; 5 , parallel longitudinal fibres of the superficial MCL.

condyle (Fig. 9). The tibial attachment was a line 2 to $3 \mathrm{~mm}$ distal to the margin of the articular cartilage, immediately proximal to the insertion of the semimembranosus tendon. The parallel fibres with a width of 5 to $9 \mathrm{~mm}$, and length of 29 to $33 \mathrm{~mm}$ ran distally and very slightly anteriorly. The deep MCL lay directly beneath the posterior parallel fibres of the superficial MCL (Fig. 6). Although a distinct structure, it was inseparable from the capsule and was also attached to the medial meniscus. The anterior edge was easily identifiable, as the deep MCL formed a distinct and easily palpable thicker band within the surrounding capsular tissue. Posteriorly, the proximal part of the ligament blended with the posteromedial capsule. The tightness of the fibres did not appear to alter significantly in flexion and extension. The fibres tightened rapidly with external tibial rotation.

The tendon of semimembranosus attached to the tibia just distal to the attachment of the deep MCL and formed a transverse oval, 10 to $13 \mathrm{~mm}$ by 4 to $5 \mathrm{~mm}$.

Posterior. Layer 1 is the fascial layer. The conjoined layers 2 and 3 formed the PMC proximal to the joint line, and the distal oblique fibres of the superficial MCL (layer 2) distal to the joint line.

PMC. The PMC had an extensive line of femoral attachment, which extended from just posterior to the attachment of the longitudinal parallel fibres around the base of the adductor tubercle in a posterior and then slightly proximal direction, passing immediately proximal to the junction 


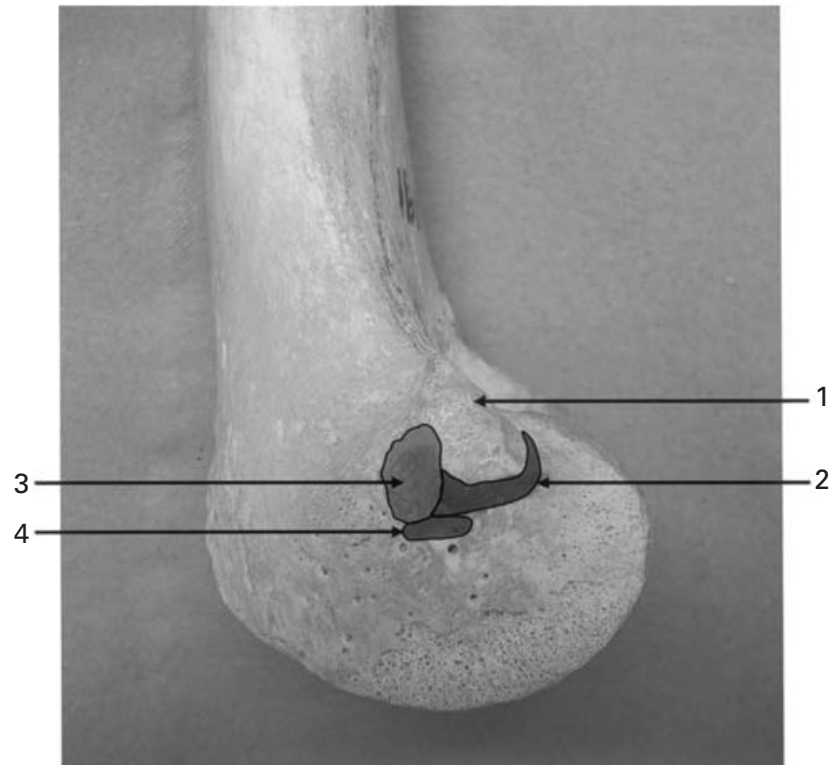

Fig. 9

Photograph of the femoral attachments of the components of the MCL complex: 1, adductor tubercle; 2, attachment of the PMC; 3 , attachment of longitudinal fibres of the superficial MCL covering epicondyle; 4 , attachment of the deep MCL.

between the bulging posterior articular surface and the shaft of the femur (Fig. 9). The fibres attached to the rim of the posteromedial aspect of the tibia, just distal to the joint line.

Proximal to the joint line, the ligamentous fibres in the posterior third could not be separated from the joint capsule. Layers 2 and 3 thereby formed the PMC. In this structure, the fibres were orientated mainly in a posterior-distal direction. The PMC was formed into a pouch which was filled and tensed by the posterior medial femoral condyle as the knee reached full extension (Fig. 5). Many of the ligamentous fibres converged towards the medial femoral epicondyle proximally. Within the expanse of the PMC, starting at the posterior margin of the longitudinal fibres of the superficial MCL and immediately distal to the adductor tubercle, there was a localised thicker band passing posterodistally from the medial femoral epicondyle, corresponding to the POL of Hughston and Eilers. ${ }^{9}$ Fibres from the semimembranosus tendon sheath blended with the PMC. Proximally, the more posterior fibres of the PMC blended with the posterior fibres of adductor magnus (Fig. $5)$.
The posteromedial capsule tightened when the knee reached full extension. It slackened almost as soon as the knee started to flex. With progressive flexion, this structure buckled and then folded, with the slacker posterior part passing deep to the more anterior (Fig. 7). Tension in the semimembranosus tendon appeared to be partly responsible for this via the attachments of the tendon sheath, which always pulled its surrounding tissues proximally. The slackness of the posteromedial capsule in flexion indicated that it did not provide a significant restraint to tibiofemoral valgus with the knee flexed. With the knee extended, internal rotation of the tibia caused the oblique fibres of the PMC to tighten further (Fig. 8). The semimembranosus tendon inserted into the tibia just distal to the tibial attachment of the deep MCL (middle third).

Distal oblique fibres of superficial MCL. The proximal attachment was to the rim of the posteromedial aspect of the medial meniscus and tibia in the distal oblique fibres of superficial MCL. Some fibres were in continuity with those of the more proximal PMC. There was no capsular layer 3 distal to this attachment. These fibres blended distally with the posterior edge of the long parallel fibres of the superficial MCL. Therefore, the tibial attachment was the same as that of the superficial MCL. The distal oblique fibres (layer 2) ran proximally and posteriorly from their distal attachment and did not traverse the joint to attach to the femur. Some of the more anterior fibres appeared to blend with fibres of the PMC as they attached to the tibia. The more posterior fibres blended with the semimembranosus tendon sheath. Because these fibres do not traverse the joint, their tension did not appear to be affected by flexion and extension. The fibres appeared to prevent the meniscus sliding posteriorly when the knee was externally rotated.

\section{Discussion}

We found that the posteromedial corner appears to have three distinct ligamentous components which cross the joint line: the superficial MCL, the deep MCL, and the PMC (Table I). The findings support the layered approach of Warren and Marshall ${ }^{5}$ and also clarify the structures anteroposteriorly by dividing the medial side into thirds. When the superficial MCL is cut just proximal to the tibial attachment it can be folded back to expose the deep MCL (Fig. 6). It is impossible to fold the layer of the superficial MCL (layer 2) back further than the proximal oblique fibres because in this region they are inseparable from layer 3 , the joint capsule. Thus in the posterior third, the super-

Table I. Simplified scheme describing the main ligamentous and capsular structures crossing the medial and posteromedial joint line

\begin{tabular}{|c|c|c|c|}
\hline & Anterior & Middle & Posterior \\
\hline Layer 1 & Layer of fascia & Layer of fascia & Layer of fascia \\
\hline $\begin{array}{l}\text { Layer } 2 \\
\text { Layer } 3\end{array}$ & $\begin{array}{l}\text { No significant ligamentous struc- } \\
\text { ture connecting femur to tibia }\end{array}$ & $\begin{array}{l}\text { Superficial MCL } \\
\text { (longitudinal fibres) } \\
\text { Deep MCL }\end{array}$ & $\left\{\begin{array}{l}\text { Posteromedial } \\
\text { capsule }\end{array}\right.$ \\
\hline
\end{tabular}


ficial and deep layers of the MCL complex blend with fibres from the tendon sheath of the semimembranosus to form what appears to be a functionally distinct structure, the PMC.

The anatomy of the posteromedial corner has been described in different ways depending upon whether it is possible to define a distinct POL as part of the posteromedial capsule. The argument among authors as to whether the oblique fibres in the posterior third are part of the superficial MCL, or a thickening of the capsule, seems immaterial. What is important is that they appear to be a functional unit with links to the semimembranosus tendon sheath. The three discrete bands of the PMC which were described by Hughston and Eilers ${ }^{9}$ could not be identified in this study. As the tibia was internally and externally rotated in extension, the orientation of different PMC fibre bands could be identified as they were co-opted to restrain movement. Some of these oblique fibres corresponded to the position of the POL described by Hughston and Eilers. ${ }^{9}$ However, we found no obviously identifiable, discrete ligamentous structure when the knee was approached from superficial to deep. Rather than a discrete ligament, there were capsular condensations, more obvious when they were tensioned while restraining movement.

Although biomechanical testing was not performed, we found that the posteromedial capsule appeared to be a passive restraint to internal rotation of the tibia with the knee in extension. As the knee progressively flexes from extension the semimembranosus tendon expansions to the PMC allow the capsule to buckle in a uniform manner. The posterior fibres of the PMC fold and are carried deep to the more anterior fibres. This slackening in the first few degrees of flexion suggests that the PMC is a passive restraint only in the extended knee.

It has been suggested that semimembranosus is a stabiliser of the medial aspect of the knee. ${ }^{9}$ Its tendon sheath may be an active restraint on the posteromedial corner when tensioned by contraction of semimembranosus. Previous authors ${ }^{10}$ have described how the PMC is "dynamised" by the semimembranosus and kept taut as the knee flexes. However, this appears to be based on a non-physiological orientation of the tendon and not confirmed by biomechanical testing. We found that because the physiological pull of the semimembranosus is approximately parallel to the axis of the femur, it only tensed the PMC at the limit of flexion. These observations suggest that the semimembranosus is aligned to act as an active restraint to valgus with the knee extended and to external rotation with the knee flexed. The tendon sheath expansions to the postero-medial corner and distal oblique fibres of the superficial MCL may act as a sling, controlling the direction of the pull of semimembranosus.

In our anatomical study, the ligament complex was assessed from the point of view of likely function. Specimens were dissected immediately after thawing, so the anatomical structures were more easily mobilised and demonstrated than in fixed specimens. Also, digital video photography helped demonstrate how the structures moved and tightened or slackened as the knee was flexed or rotated. Photographs presenting the results have avoided errors of interpretation which arise in the production of a drawing.

We aimed to reconcile the different published descriptions and to present an interpretation of the anatomy that is useful for surgeons. This initial study is the basis for further work to evaluate how these ligamentous structures act as passive restraints to tibiofemoral kinematics. In particular, it has led to questions about how the semimembranosus may act as a dynamic stabiliser. The availability of further biomechanical evidence of the function of the structures described here may improve the diagnosis and surgical treatment of patients with complex knee ligament injuries.

Mr J. Robinson and Mr J. Sanchez-Ballester held the post of Clinical Research Fellow in Sports and Trauma Surgery at Imperial College. The post was part funded by Ealing Hospital NHS Trust. Dr A. Bull is funded by the Arthritis Research Campaign.

We thank the Pathology Department and the Patient Affairs Office at Ealing Hospital for their help.

No benefits in any form have been received or will be received from a commercial party related directly or indirectly to the subject of this article.

\section{References}

1. Jarvinen M, Natri A, Laurila S, Kannus P. Mechanisms of anterior cruciate ligament ruptures in skiing. Knee Surg Sports Traumatol Arthrosc 1994;2:224-8.

2. Friden T, Erlandsson T, Zatterstrom R, Lindstrand A, Moritz U. Compression or distraction of the anterior cruciate injured knee: variations in injury pattern in contact sports and downhill skiing. Knee Surg Sports Traumatol Arthrosc 1995;3:144-7.

3. Palmer I. On the injuries to the ligaments of the knee joint. Acta Orthop Scand Suppl 1938:5-283

4. Brantigan OC, Voshell AF. The tibial collateral ligament: its function, its bursae, and its relation to the medial meniscus. J Bone Joint Surg 1943;25:121-31.

5. Warren LF, Marshall JL. The supporting structures and layers on the medial side of the knee: an anatomical analysis. J Bone Joint Surg [Am] 1979;61-A:56-62.

6. Mains DB, Andrews JG, Stonecipher T. Medial and anterior-posterior ligament stability of the human knee, measured with a stress apparatus. Am J Sports Med 1977:5:144-53.

7. Last RJ. Some anatomical details of the knee-joint. J Bone Joint Surg [Br] 1948; 30-B: 683-8.

8. Williams P, Warwick R, Dyson M, et al. Gray's anatomy. 38th ed. Edinburgh: Churchill Livingstone, 1995

9. Hughston JC, Eilers AF. The role of the posterior oblique ligament in repairs of acute medial (collateral) ligament tears of the knee. J Bone Joint Surg [Am] 1973; 55-A:923-40.

10. Strobel M, Stedfeld H-W. Diagnostic evaluation of the knee. Berlin:Springer-Verlag, 1990:17-25

11. Warren LF, Marshall JL, Girgis F. The prime static stabiliser of the medial side of the knee. J Bone Joint Surg [Am] 1974;56-A:665-74.

12. Slocum DB, Larson RL. Rotary instability of the knee: its pathogenesis and a clinical test to determine its presence. J Bone Joint Surg [Am] 1968;50-A:211-25

13. Horwitz MT. An investigation of the surgical anatomy of the ligaments of the knee joint. Surg Gynec and Ost 1938;57:287-91.

14. Policau Prose L, Lohman AHM, Huson A. The collateral ligaments of the knee joint in the cat and man. Acta Anat (Basel) 1988;133:70-8.

15. Kennedy JC, Fowler PJ. Medial and anterior instability of the knee. J Bone Joint Surg $[$ Am] 1971;53-A:1257-70.

16. Nielsen S, Kromann Andersen C, Rasmussen 0, Andersen K. Instability of cadaver knees after transection of capsule and ligaments. Acta Orthop Scand 1984; 55:30-4.

17. Fischer RA, Arms SW, Johnson RJ, Pope MH. The functional relationship of the posterior oblique ligament to the medial collateral ligament of the human knee. Am J Sports Med 1985;13:390-7.

18. Kaplan EB. Some aspects of functional anatomy of the human knee joint. Clin Orthop $1961 ; 23: 18-29$. 